University of Nebraska - Lincoln

DigitalCommons@University of Nebraska - Lincoln

Faculty Publications from the Department of Engineering Mechanics

Mechanical \& Materials Engineering,

Department of

$9-2002$

\title{
Closed-form solution for the size of plastic zone in an edge- cracked strip
}

Xiang-Fa Wu

University of Nebraska-Lincoln, xfwu@unlserve.unl.edu

Yuris A. Dzenis

University of Nebraska - Lincoln, ydzenis@unl.edu

Follow this and additional works at: https://digitalcommons.unl.edu/engineeringmechanicsfacpub

Part of the Mechanical Engineering Commons

Wu, Xiang-Fa and Dzenis, Yuris A., "Closed-form solution for the size of plastic zone in an edge-cracked strip" (2002). Faculty Publications from the Department of Engineering Mechanics. 80.

https://digitalcommons.unl.edu/engineeringmechanicsfacpub/80

This Article is brought to you for free and open access by the Mechanical \& Materials Engineering, Department of at DigitalCommons@University of Nebraska - Lincoln. It has been accepted for inclusion in Faculty Publications from the Department of Engineering Mechanics by an authorized administrator of DigitalCommons@University of Nebraska - Lincoln. 
Published in International Journal of Engineering Science 40:15 (September 2002), pp. 1751-1759;

doi: 10.1016/S0020-7225(02)00031-9 http://www.elsevier.com/wps/product/cws_home/278

Copyright ( 2002 Elsevier Science Ltd. Used by permission.

Submitted November 16, 2001; revised February 20, 2002; accepted March 20, 2002; published online June 3, 2002.

\title{
Closed-form solution for the size of plastic zone in an edge-cracked strip
}

\author{
Xiang-Fa Wu and Yuris A. Dzenis \\ Department of Engineering Mechanics, Center for Materials Research \& Analysis, \\ University of Nebraska-Lincoln, Lincoln, NE 68588-0526, USA \\ Corresponding author - X.-F. Wu, fax 402 472-8292, email xfwu@unlserve.unl.edu or wuxiangfa@yahoo.com
}

\begin{abstract}
This paper is concerned with the problem of plastic zone at the tip of an edge crack in an isotropic elastoplastic strip under anti-plane deformations. By means of complex potential and Dugdale model, the stress intensity factor and the size of plastic zone are obtained in closed-form. Furthermore, the analytic solutions for an edge crack at the free boundary of a half-space and a semi-infinite crack heading towards a free surface are determined as the limiting cases of the strip geometries.
\end{abstract}

Keywords: edge crack, plastic zone size, strip, stress intensity factor

\section{Introduction}

Crack tip opening displacement and the size of plastic zone near crack tips are two important fundamental parameters utilized in nonlinear analysis of cracks in ductile materials. Based on the assumption of constant cohesive stress at the leading edge of the crack, the Dugdale model was utilized extensively for investigating the plastic deformation near crack tips, and yielded reliable predictions at the ends of stationary slits in steel sheets [1]. The Dugdale model provides a feasible method to estimate the size of the plastic zone near crack tip in the framework of linear fracture mechanics.

A number of solutions for notches, cracks and spatial penny-shaped cracks under anti-plane or inplane deformations were obtained using the Dugdale hypothesis, for example, see recent works by 
Singh and coworkers [2-10], Olesiak and Wnuk [11], Olesiak and Shadley [12], Tsai [13], Fan [14, 15], Wang and Shen [16]. It is also important to mention the early work by Atkinson and Howard [17], Bilby et al. [18], Field [19], Koskinen [20], Rice [21], and Smith [22]. A brief literature review on the Dugdale model and its related applications can be found in the recent paper by Vrbik et al. [10].

In the present work, on the basis of complex displacement potential and Dugdale model, a method is proposed to find the closed-form solutions for the stress intensity factor (SIF) and the size of the plastic zone of an edge crack in an isotropic elastoplastic strip under anti-plane deformations. The solution is obtained by introducing a conformal mapping, which maps the edge-cracked strip onto the whole plane with a semi-infinite cut. Compared with the methods developed in literature, the current method provides a more concise way to extract the closed-form solutions.

As the limiting cases of the current problem, the SIF and plastic zone size solutions for an edge crack at the free boundary of a half-plane as well as a semi-infinite crack heading towards a free surface can be determined directly without recourse to the method of dual integral equations or the Wiener-Hopf technique.

\section{Formulation and solution procedure}

Cracks in elastic media under deformations can be analysed as interactions between a dislocation and cracks. In the anti-plane case, the displacement and stress components of an isotropic elastic body can be expressed in terms of an analytic function $\phi(\zeta)$ as follows:

$$
u_{3}(\xi, \eta)=-\frac{2}{\mu} \operatorname{Im}[\varphi(\zeta)], \quad \sigma_{23}(\xi, \eta)=-2 \operatorname{Re}\left[\varphi^{\prime}(\zeta)\right], \quad \sigma_{13}(\xi, \eta)=2 \operatorname{Re}\left[\mathrm{i} \varphi^{\prime}(\zeta)\right]
$$

where the prime (') denotes the derivative with respect to $\zeta=\xi+i \eta$, and $\mu$ is the material shear modulus.

Consider a semi-infinite crack interacting with a singularity (a screw dislocation or a line force) in an infinite plane as shown in Figure 1(a). The singularity is located at $\zeta_{0}=\xi_{0}+i \eta_{0}$ where $\xi$ and $\eta$ are the coordinates of the material points, $(\xi, \eta)$ with the origin located at the crack tip. Thomson [23] obtained the complex displacement potential for this anti-plane problem as follows:

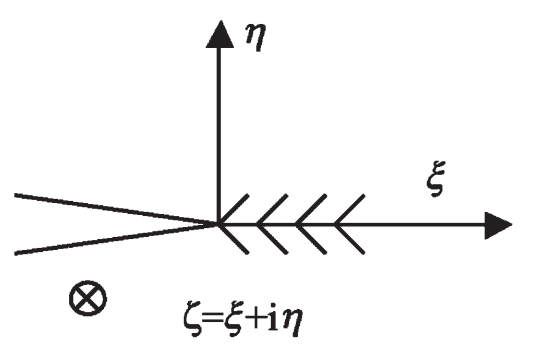

$\left(\xi_{0}, \eta_{0}\right) \quad(a)$

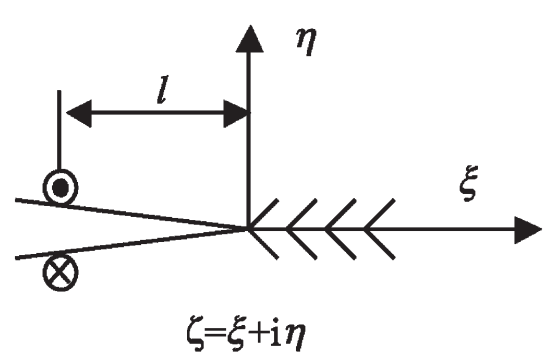

(b)

Figure 1. A semi-infinite crack in the whole space under anti-plane deformations: (a) with an anti-plane singularity and (b) with a pair of concentrated forces located at crack surfaces. 


$$
\varphi^{\prime}(\zeta)=\frac{q}{\zeta-\zeta_{0}}-\frac{q}{2 \sqrt{\zeta}}\left(\frac{q}{\sqrt{\zeta}+\sqrt{\zeta_{0}}}+\frac{\bar{q}}{\sqrt{\zeta}+\sqrt{\zeta_{0}}}\right)
$$

where the over bar $\left(^{-}\right)$denotes the complex conjugate.

Here, the quantity $q$ is defined as

$$
q=-\frac{b \mu}{4 \pi}+i \frac{p}{4 \pi}
$$

in which $b$ is the Burgers vector of the screw dislocation and $p$ is the line force.

The SIF $\left(K_{\mathrm{III}}\right)$ and the energy release rate $(\mathrm{ERR})\left(G_{\mathrm{III}}\right)$ are evaluated respectively as

$$
K_{\mathrm{III}}=\lim _{\xi \rightarrow 0}-2 \sqrt{2 \pi \xi} \varphi^{\prime}(\xi), \quad G_{\mathrm{III}}=\frac{K_{\mathrm{III}}^{2}}{2 \mu} .
$$

Under the action of a singularity $q$ at $\zeta_{0}$, substitution of (2) and (3) into (4) yields

$$
K_{\mathrm{III}}=\sqrt{2 \pi}\left(\frac{q}{\sqrt{\zeta_{0}}}+\frac{\bar{q}}{\sqrt{\bar{\zeta}_{0}}}\right)
$$

When the crack is opened by a pair of self-equilibrated anti-plane forces $P_{0}$ acting on the crack surfaces a distance $l$ behind the crack tip, the corresponding SIF and ERR may be extracted from (4) and (5) as

$$
K_{\mathrm{III}}=\frac{2 P_{0}}{\sqrt{2 \pi l}}, \quad G_{\mathrm{III}}=\frac{P_{0}^{2}}{\pi \mu l} .
$$

Let us now consider an edge crack at the free boundary of a strip with an anti-plane singularity (a screw dislocation or a line force) located at $z_{0}=x_{0}+\mathrm{i} y_{0}$ as shown in Figure 2(a). Here, $a$ and $W$ denote the crack length and the strip width, respectively. Consider the conformal mapping,

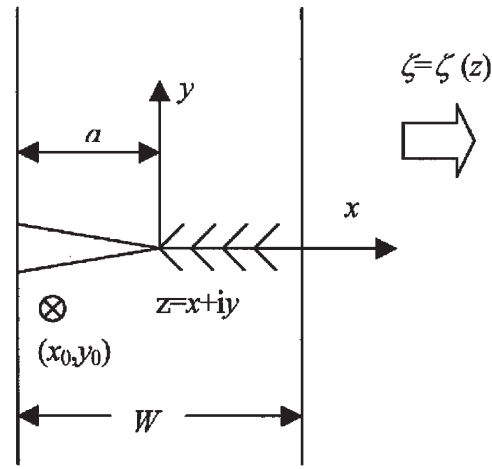

(a)

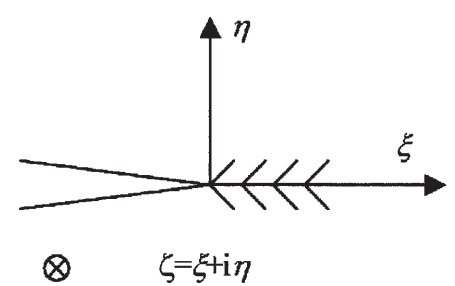

$\left(\xi_{0}, \eta_{0}\right)$

Figure 2. Cracked-material with an anti-plane singularity: (a) an edge-cracked strip (z-plane) and (b) a semi-infinite crack in the whole plane ( $\zeta$-plane). 


$$
\zeta=\left[\frac{\operatorname{tg}(Z+A)}{\operatorname{tg}(A)}\right]^{2}-1
$$

with

$$
Z=\frac{\pi z}{2 W}=\frac{\pi(x+\mathrm{i} y)}{2 W}, \quad A=\frac{\pi a}{2 W}
$$

which maps the edge-cracked strip onto the whole plane with a semi-infinite cut along its negative $\xi$-axis as shown in Figure 2(b). Substituting (7) into (2), we obtain the complex potential of the edgecracked strip in the $z$-plane as

$$
\varphi^{\prime}(z)=\frac{\pi}{W} \operatorname{tg}(Z+A) \sec ^{2}(Z+A)\left\{q g_{1}(Z)-\frac{q g_{2}(Z)+\bar{q} g_{3}(Z)}{2 \sqrt{\operatorname{tg}^{2}(Z+A)-\operatorname{tg}^{2}(A)}}\right\},
$$

where

$$
\begin{aligned}
& g_{1}(Z)=\frac{1}{\operatorname{tg}^{2}(Z+A)-\operatorname{tg}^{2}\left(Z_{0}+A\right)}, \\
& g_{2}(Z)=\frac{1}{\sqrt{\operatorname{tg}^{2}(Z+A)-\operatorname{tg}^{2}(A)}+\sqrt{\operatorname{tg}^{2}\left(Z_{0}+A\right)-\operatorname{tg}^{2}(A)}}, \\
& g_{3}(Z)=\frac{1}{\sqrt{\operatorname{tg}^{2}(Z+A)-\operatorname{tg}^{2}(A)}+\sqrt{\overline{\operatorname{tg}^{2}\left(Z_{0}+A\right)}-\operatorname{tg}^{2}(A)}},
\end{aligned}
$$

$Z$ and $A$ are defined in (8), and $q$ is the singularity quantity defined in (3).

Substitution of (9)-(12) into (4) yields the SIF, which can be used as the Green's function to calculate the SIF and ERR of an edge-cracked strip under arbitrary anti-plane deformations.

Now consider the SIF for the edge crack in an isotropic strip opened by a pair of self-equilibrated anti-plane forces, $P_{0}$ at the crack surfaces a distance $l$ behind the crack tip, as shown in Figure 3(a). Substituting (9) into (4), we obtain the SIF as

$$
\begin{aligned}
K_{\mathrm{III}} & =\lim _{x \rightarrow 0}-2 \sqrt{2 \pi x} \varphi_{1}^{\prime}(x)=\lim _{x \rightarrow 0}-4 \sqrt{2 \pi x} \varphi^{\prime}(x) \\
& =\frac{2 P_{0}}{\sqrt{\pi a}} \sec \left(\frac{\pi a}{2 W}\right) \sqrt{\frac{\pi a}{2 W} \operatorname{tg} \frac{\pi a}{2 W} /\left(\operatorname{tg}^{2} \frac{\pi a}{2 W}-\operatorname{tg}^{2} \frac{\pi(a-l)}{2 W}\right)} .
\end{aligned}
$$

Furthermore, as shown in Figure 3(b), using (13) as the Green's function, we may obtain the SIF for the strip with crack surfaces under the action of self-equilibrated forces $p(x)$ applied in the interval $x \in$ $[-l, 0]$ as 


$$
K_{\mathrm{III}}=\frac{2 \sec (\pi a / 2 W)}{\sqrt{\pi a}} \sqrt{\frac{\pi a}{2 W} \operatorname{tg} \frac{\pi a}{2 W}} \int_{0}^{l} \frac{p(x)}{\sqrt{\operatorname{tg}^{2}(\pi a / 2 W)-\operatorname{tg}^{2}(\pi(a-x) / 2 W)}} \mathrm{d} x
$$

When uniform forces are considered, say $p(x)=p_{0}$, relation (14) reduces to

$$
K_{\mathrm{III}}=p_{0} \sqrt{\pi a} \sqrt{\frac{2 W}{\pi a} \operatorname{tg} \frac{\pi a}{2 W}}\left\{1-\frac{2}{\pi} \arcsin \left\{\frac{\sin [\pi(a-l) / 2 W]}{\sin (\pi a / 2 W)}\right\}\right\} .
$$

Setting $l=a$, relation $(15)$ becomes

$$
K_{\mathrm{III}}=p_{0} \sqrt{\pi a} \sqrt{\frac{2 W}{\pi a} \operatorname{tg} \frac{\pi a}{2 W}}
$$

which agrees with the result derived by means of Westergaard stress function [24],

$$
Z(z)=p_{0} \sin \left(\frac{\pi z}{2 W}\right) / \sqrt{\sin ^{2}\left(\frac{\pi z}{2 W}\right)-\sin ^{2}\left(\frac{\pi a}{2 W}\right)} .
$$

Under anti-plane deformations, the discussed edge-cracked strip is mathematically equivalent to collinear periodic cracks in an infinite space.

Now consider the plastic zone near the edge crack tip in the strip with uniform forces, $p_{0}$ acting on the crack surfaces. The plastic zone is described by line $0<x<d$ as shown in Figure 3(b). On the basis of the Dugdale hypothesis [1], the shear stress in the yield zone is equal to a constant yield stress, $Y$, and the singularity of shear stress at the leading of the crack $(x=d)$ is removed. Utilizing (13) as the Green's function for the current problem, we obtain the size of the plastic zone by solving the following integral equation:

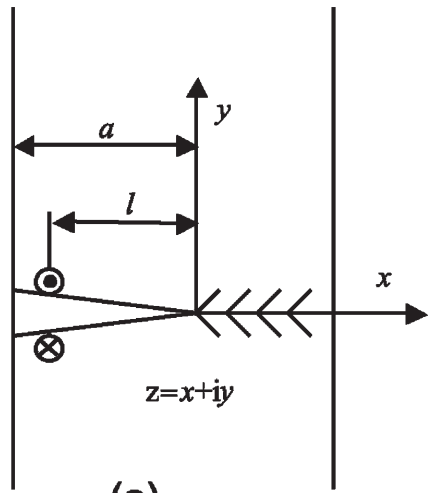

(a)

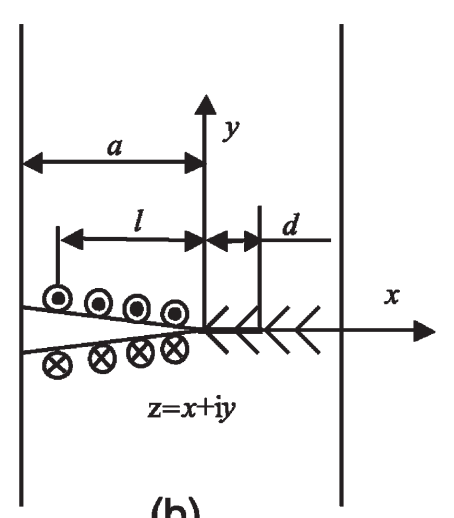

(b)

Figure 3. An edge-cracked strip under anti-plane deformations: (a) a pair of concentrated forces located at crack surfaces and (b) uniform forces at crack surfaces. 


$$
\begin{aligned}
& \int_{d}^{d+l} \frac{p_{0}}{\sqrt{\operatorname{tg}^{2}(\pi(a+d) / 2 W)-\operatorname{tg}^{2}(\pi(a+d-x) / 2 W)}} \mathrm{d} x \\
& \quad=\int_{0}^{d} \frac{Y}{\sqrt{\operatorname{tg}^{2}(\pi(a+d) / 2 W)-\operatorname{tg}^{2}(\pi(a+d-x) / 2 W)}} \mathrm{d} x
\end{aligned}
$$

which yields

$$
\arcsin \left\{\frac{\sin (\pi a / 2 W)}{\sin [\pi(a+d) / 2 W]}\right\}=\frac{\pi}{2(1+\lambda)}\left\{1+\frac{2 \lambda}{\pi} \arcsin \left\{\frac{\sin [\pi(a-l) / 2 W]}{\sin [\pi(a+d) / 2 W]}\right\}\right\},
$$

where

$$
\lambda=\frac{p_{0}}{Y}
$$

Setting $l=a$ in (18), we obtain the plastic zone solution for uniform forces at the crack surfaces as

$$
\arcsin \left\{\frac{\sin (\pi a / 2 W)}{\sin [\pi(a+d) / 2 W]}\right\}=\frac{\pi}{2(1+\lambda)} .
$$

Consequently, letting $d=W-a$ in (20), we obtain the limiting uniform force $p_{0}$ at which the strip fully yields along the crack line across the strip as

$$
p_{0}=\left(\frac{W}{a}-1\right) Y .
$$

The explicit relation (20) determines the plastic zone size near the edge crack tip in an isotropic elastoplastic strip. Vrbik et al. [10] provided a numerical solution of the same problem with the help of the Fredholm integrals of the second kind. The current results are compared with the results of [10] in Figure 4. It is evident that the two methods predict similar trends. Since the present solution (20) is

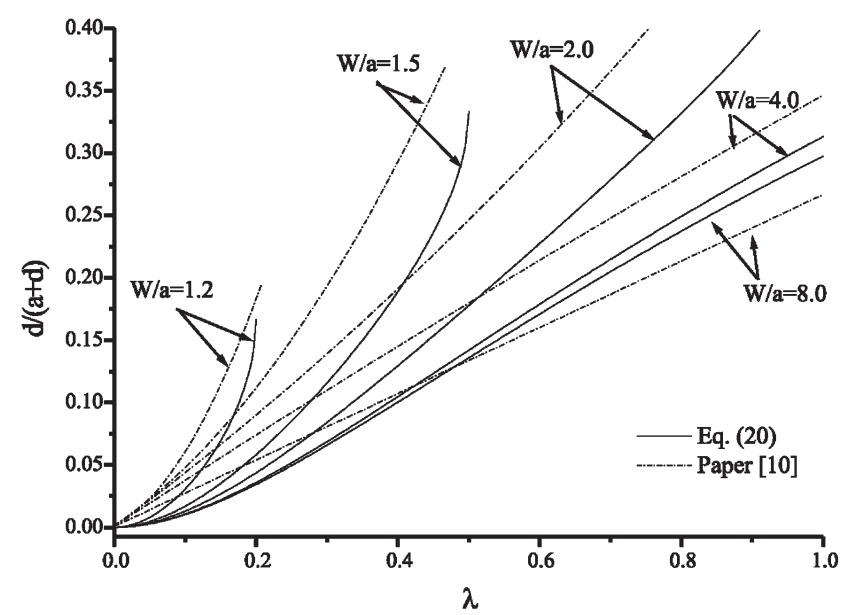

Figure 4. Variation of plastic zone size with $\lambda$ for different values of $W / a=1.2,1.5,2,3,8$. 
an explicit one, the deviation between the two approaches may be caused by the numerical procedure in [10].

\section{Examples and discussion}

Here we consider the SIF and plastic zone size solutions for an edge crack at the free boundary of a half-space and a semi-infinite crack heading towards a free surface etc.

As shown in Figure 5(a) and (b), letting $W \rightarrow \infty$ in (13) and (15), we obtain the SIFs for the edge crack at the free boundary of a half-space as

$$
K_{\mathrm{III}}=\frac{2 P_{0}}{\sqrt{\pi a}} \frac{a}{\sqrt{a^{2}-(a-l)^{2}}}
$$

and

$$
K_{\mathrm{III}}=p_{0} \sqrt{\pi a}\left[1-\frac{2}{\pi} \arcsin \left(1-\frac{l}{a}\right)\right] .
$$

Setting $l=a$, relation $(23)$ reduces to

$$
K_{\mathrm{III}}=p_{0} \sqrt{\pi a}
$$

which is the solution for uniform forces acting on the entire crack surfaces.

Alternatively, we may derive relations (22)-(24) by letting $W \rightarrow \infty$ in (7) and using the conformal mapping function, $\zeta=(z / a-1)^{2}-1$. Furthermore, as shown in Figure 6(a) and (b), relations (22) and (24) are exactly the SIFs for a Griffith crack in an infinite space with two pairs of self-equilibrated forces, $P_{0}$, and uniform anti-plane forces, $p_{0}$ at the crack surfaces, respectively.
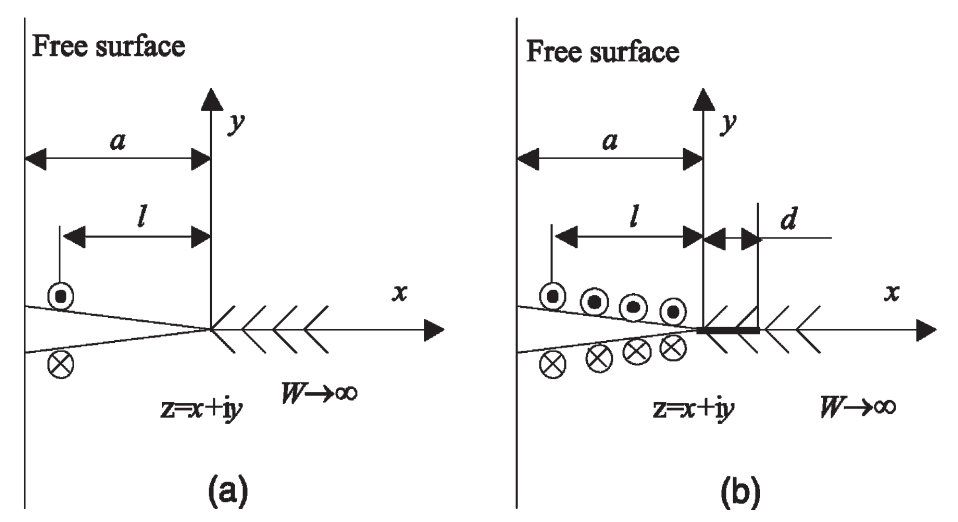

Figure 5. An edge-cracked half space under anti-plane deformations: (a) a pair of concentrated forces located at crack surfaces and (b) uniform forces at crack surfaces. 

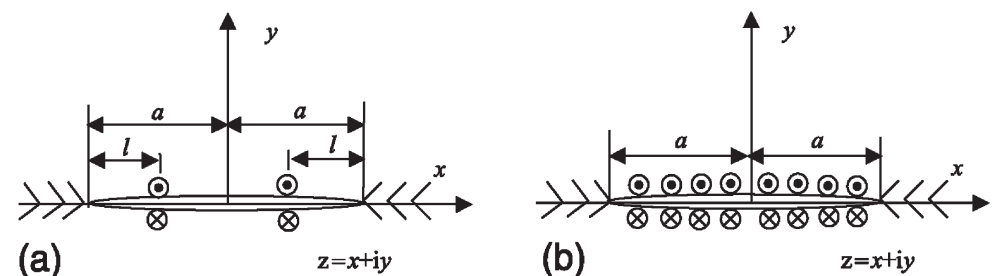

(b)

$\mathrm{z}=x+\mathrm{i} y$

Figure 6. A Griffith crack in the whole space under anti-plane deformations: (a) two pairs of concentrated forces located at crack surfaces and (b) uniform forces at crack surfaces.

Letting $W \rightarrow \infty$ in (18), we obtain the corresponding plastic zone size as

$$
\arcsin \left(\frac{a}{a+d}\right)=\frac{\pi}{2(1+\lambda)}\left\{1+\frac{2 \lambda}{\pi} \arcsin \left(\frac{a-l}{a+d}\right)\right\} .
$$

Furthermore, setting $l=a$, relation (25) becomes

$$
\arcsin \left(\frac{a}{a+d}\right)=\frac{\pi}{2(1+\lambda)},
$$

which accords with the result derived by the method of dual integral equations [10].

As shown in Figure 7(a) and (b), letting $a \rightarrow \infty$ and simultaneously keeping $c=(W-a)$ constant in (13) and (15), we obtain the SIFs for a semi-infinite crack heading towards a free surface as

$$
K_{\mathrm{III}}=\frac{2 P_{0}}{\sqrt{\pi l}} \frac{l+c}{\sqrt{c(l+2 c)}}
$$

and

$$
K_{\mathrm{III}}=2 p_{0} \sqrt{\frac{l(l+2 c)}{\pi c}} .
$$

Consequently, letting $c \rightarrow \infty$ relation (27) returns to (6), and (28) becomes

$$
K_{\mathrm{III}}=\frac{2 \sqrt{2}}{\pi} p_{0} \sqrt{\pi l}
$$

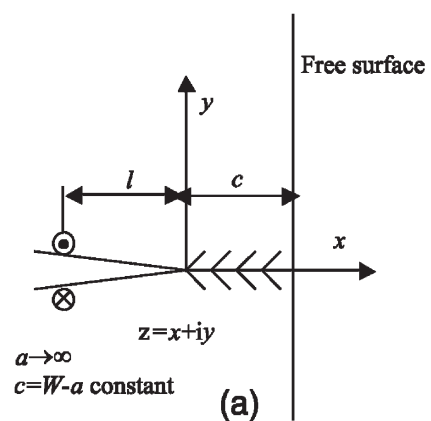

(a)

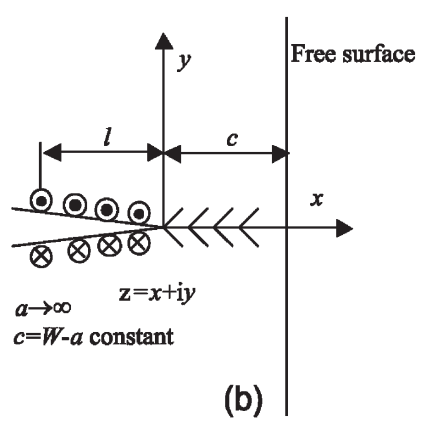

Figure 7. A semi-infinite crack in a half space under anti-plane deformations: (a) a pair of concentrated forces located at crack surfaces and (b) uniform forces at crack surfaces. 
which is the solution for a semi-infinite crack in the whole space with uniform forces, $p_{0}$ on the crack surfaces, $0<x<l$.

Acknowledgments - The support of this work by the US Army Research Office is gratefully acknowledged. The authors would like to thank the anonymous reviewers for International Journal of Engineering Science for their helpful suggestions to improve this paper.

\section{References}

1. D. S. Dugdale, J. Mech. Phys. Solids 8 (1960), pp. 100-104.

2. B. M. Singh, H. T. Danyluk, and J. Vrbik, Acta Mech. 55 (1985), pp. 81-86.

3. H. T. Danyluk and B.M. Singh, Acta Mech. 56 (1985), pp. 75-92.

4. B. M. Singh, H. T. Danyluk, J. Vrbik, and A.P.S. Selvadurai, Eng. Fract. Mech. 24 (1986), pp. 39-44.

5. B. M. Singh, A. Cardou, H. T. Danyluk, and J. Vrbik, Theor. Appl. Fract. Mech. 8 (1987), pp. 193-197.

6. B. M. Singh, A. Cardou, and M. C. Au, Eng. Fract. Mech. 29 (1988), pp. 500-511.

7. H. T. Danyluk, B. M. Singh, and J. Vrbik, Int. J. Fract. 51 (1991), pp. 331-342.

8. H. T. Danyluk, B. M. Singh, and J. Vrbik, Eng. Fract. Mech. 51 (1995), pp. 735-740.

9. H. T. Danyluk, B. M. Singh, and J. Vrbik, Int. J. Fract. 75 (1996), pp. 307-322.

10. J. Vrbik, B. M. Singh, J. Rokne, and R.S. Dhaliwal, Z. Angew. Math. Mech. (ZAMM) 81 (2001), pp. 642-647.

11. Z. Olesiak and M. Wnuk, Int. J. Fract. Mech. 4 (1968), pp. 383-385.

12. Z. Olesiak and J. R. Shadley, Int. J. Fract. Mech. 5 (1969), pp. 305-313.

13. Y. M. Tsai, Int. J. Mech. Sci. 26 (1984), pp. 245-252.

14. T. Y. Fan, Eng. Fract. Mech. 37 (1990), pp. 1085-1087.

15. T. Y. Fan, Eng. Fract. Mech. 44 (1993), pp. 243-246.

16. X. M. Wang and Y. P. Shen, Int. J. Fract. 59 (1993), pp. R25-R32.

17. C. Atkinson and I. C. Howard, Int. J. Fract. Mech. 6 (1970), pp. 96-97.

18. B. A. Bibly, A. H. Cottrell, and K. H. Swinden, Proc. Roy. Soc.: Ser. A 272 (1963), pp. 304-314.

19. F. A. Field, J. Appl. Mech. 30 (1963), pp. 622-623.

20. M. F. Koskinen, J. Basic Eng. Trans. ASME 85 (1963), pp. 585-594.

21. J. R. Rice, in Proceedings of 1st International Conference in Fracture, Sendi, Japan (1965), p. 309.

22. E. Smith, Int. J. Eng. Sci. 5 (1967), pp. 791-799.

23. R. Thomson, Solid State Phys. 39 (1986), pp. 1-129.

24. H. Tada, P. C. Paris, and G. R. Irwin, The Stress Analysis of Cracks Handbook, Del Research, Hellertown, PA (1973). 